\section{Pai pobre, filho pobre? Uma análise da mobilidade intergeracional de renda na coorte de nascimentos de 1982, Pelotas, Rio Grande do Sul, Brasil}

\author{
Poor dad, poor child? An investigation of \\ intergenerational income mobility in the 1982 Birth \\ Cohort in Pelotas, Rio Grande do Sul State, Brazil
}

¿Padre pobre, hijo pobre? Un análisis de la movilidad intergeneracional de ingresos en la cohorte de nacimientos de 1982, Pelotas, Rio Grande do Sul, Brasil

\begin{abstract}
Brazil is one of the countries with the lowest intergenerational income mobility. This article aimed to analyze intergenerational income mobility in the 1982 Birth Cohort in Pelotas, Rio Grande do Sul State. Two methods were used, intergenerational income elasticity and quantile regressions, in order to measure heterogeneity in income mobility as a function of different levels of parents' past income. The results show relatively high income mobility for Brazilian standards. The main explanation is that the data cover the children's income at a younger age (about 23 years). Quantile regressions show higher social mobility in the intermediary social stratum. The results reinforce the notion of two opposite "traps", poverty and wealth.
\end{abstract}

Social Class; Income; Cohort Studies
Cesar Augusto Oviedo Tejada 1

Andréa D. Bertoldi 2

André Carraro 1

Felipe Garcia Ribeiro 1

Janaína Vieira dos Santos Motta 2,3

Fernando Celso Barros 2

Bernardo Lessa Horta 2

Aluísio J. D. Barros 2

\section{Resumo}

O Brasil é apontado como um dos países com as menores mobilidades intergeracionais de renda do mundo. O principal objetivo do artigo é analisar a mobilidade intergeracional de renda na coorte de nascimentos de 1982 em Pelotas, Rio Grande do Sul. São utilizados dois métodos de estimação: elasticidade intergeracional de renda e regressões quantílicas para mensuração de possíveis heterogeneidades na mobilidade intergeracional em função dos diferentes níveis de renda dos pais no passado. Os resultados mostram uma mobilidade intergeracional de renda relativamente alta para os padrões brasileiros. A explicação reside, sobretudo, no fato de terem sido usados dados de renda dos filhos em idade jovem, em torno de 23 anos. As análises das regressões quantílicas indicam que a maior mobilidade social acontece nas camadas sociais intermediárias. Os resultados encontrados reforçam a existência de dois polos opostos de armadilhas: armadilha da pobreza e da riqueza.

Classe Social; Renda; Estudos de Coortes 


\section{Introdução}

O tamanho da classe média na América Latina e no Caribe aumentou em 50\% entre 2003 e 2009 (de 103 milhões a 152 milhões de pessoas), representando $30 \%$ da população da região em 2009. No mesmo período, conforme aumentava a renda das famílias e diminuía a desigualdade na maioria dos países, a proporção de pessoas em estado de pobreza diminuiu significativamente de $44 \%$ para $30 \% 1$. Essas grandes mudanças no tamanho e composição das classes sociais resultaram em uma substancial mobilidade socioeconômica. Um grande número de pessoas que era pobre na década de 1990 não é mais pobre. Outros que ainda não eram de classe média já se tornaram parte dela 1 .

A mobilidade intergeracional socioeconômica refere-se à relação entre a posição socioeconômica dos pais e a posição socioeconômica dos seus filhos quando se tornam adultos. Ela avalia o grau em que a posição socioeconômica das pessoas em uma geração depende da posição socioeconômica dos seus pais. Em outras palavras, reflete o grau em que os indivíduos se deslocam para cima ou para baixo na escala social em comparação com seus pais. Ela tem sido analisada na literatura pelo uso de variáveis como a renda, a educação e a ocupação 2,3.

A mobilidade intergeracional da renda é importante por vários motivos: (a) está associada com a desigualdade da renda - países com alta desigualdade de renda e baixa mobilidade intergeracional perpetuam essa desigualdade; (b) tem forte associação com a perpetuação da pobreza, e a baixa mobilidade propicia uma transmissão intergeracional da pobreza e; (c) para alguns é considerada um possível indicador da igualdade de oportunidades econômicas em uma sociedade, em diversos contextos, pois uma menor mobilidade intergeracional pode implicar desigualdade de oportunidades 3,4 .

Os mecanismos pelos quais as rendas são transmitidas intergeracionalmente podem ser explicados teoricamente. Quanto maior o investimento privado dos pais no capital humano dos filhos, quanto maior o rendimento para qualquer investimento, quanto maior o grau de transmissão de outros aspectos do background familiar importantes para os rendimentos e quanto menos progressivo o investimento público em capital humano dos filhos, maior será o grau de transmissão intergeracional de renda 5,6.

Um número cada vez maior de estudos investiga o grau de mobilidade intergeracional e o conceito relacionado de grau de transmissão (ou de persistência) intergeracional de renda entre pais e filhos 3,7 . As pesquisas mostram grandes diferenças no grau de persistência social 2,4,8. Em alguns países desenvolvidos, como Estados Unidos, Reino Unido, Itália e Japão, mostrou-se que a persistência socioeconômica é alta 9,10,11,12 .

Comparações internacionais colocam o Brasil entre aqueles com menor mobilidade intergeracional de renda do mundo 13. Estudos analisando apenas o caso do Brasil também apresentam evidência de baixa mobilidade 14,15,16 . Em comum, esses trabalhos utilizaram dados da Pesquisa Nacional por Amostra de Domicílios (PNAD) de 1996, que possui informação de renda do filho, mas não traz informações sobre a renda dos pais. Para suprir essa deficiência, cada trabalho utiliza alguma técnica estatística para inferir o salário do pai, baseando-se em suas características de educação e ocupação.

O presente estudo tem por objetivo estimar a mobilidade intergeracional de renda na coorte de nascimentos de 1982 em Pelotas, Rio Grande do Sul, Brasil, utilizando dados coletados ao longo de 22 anos de acompanhamento dessa coorte.

\section{Métodos}

A coorte de nascimentos de 1982 recrutou 5.914 crianças nascidas vivas no Município de Pelotas, durante o ano de 1982. No acompanhamento de 1984, quando as crianças tinham dois anos, foram entrevistadas 4.934 crianças e, no acompanhamento de 2004/2005, aos 22-23 anos, foram entrevistadas 4.297 (73\% da coorte original). Como a renda dos pais, em valores nominais, foi informada apenas no acompanhamento de 1984, utilizaram-se as informações desse acompanhamento. A renda utilizada é a renda do chefe da família (que representa, em média, $86 \%$ da renda familiar na amostra). A renda dos filhos é referente ao acompanhamento realizado nos anos 2004/2005, quando estes tinham 22,7 anos, em média. As entrevistas em 1984, bem como as de $2004 / 2005$, foram realizadas ao longo de diferentes meses do ano, e os valores de renda estão fortemente influenciados pela presença de inflação e mudança de unidade monetária. Como forma de corrigir o impacto da inflação nos valores monetários, todos os valores de renda de pais e filhos foram atualizados para Reais de novembro de 2005. A escolha desse mês deve-se ao fato de ele ser o último mês do acompanhamento de $2004 / 2005$. O procedimento de cálculo dos valores da renda do país para Reais de novembro de 2005 envolveu o aplicativo calculadora do cidadão, disponibilizado pelo Banco Central em seu sítio (Banco Central do Brasil. Calculadora do cidadã: correção de valores. https://www3.bcb. gov.br/CALCIDADAO/publico/exibirFormCor 
recaoValores.do?method=exibirFormCorrecao Valores, acessado em 20/Set/2014) O índice de preços escolhido foi o Índice Nacional de Preços ao Consumidor Amplo (IPCA).

Outros critérios de seleção foram aplicados para se chegar à amostra final usada na estimação. Dos 4.297 filhos entrevistados em 2004/2005, 2.782 (64\% do total), declararam que trabalhavam e, portanto, tinham alguma renda própria. Desses, foram excluídos aqueles que declararam que não tinham informações sobre a renda do chefe da família, resultando uma amostra de 1.393 indivíduos. Finalmente, foram excluídos os indivíduos que relataram rendas extremamente baixas (rendas inferiores a $25 \%$ do salário mínimo que em novembro de 2005 era de $\mathrm{R} \$ 300,00$ ). A amostra final ficou composta por 1.133 pares de pais/filhos.

Para a análise de mobilidade, duas abordagens metodológicas foram utilizadas: análise de regressão e regressões quantílicas. Foi utilizado um modelo de regressão linear na forma: $y_{i}=\alpha+\beta x_{i}+\varepsilon_{i}$ onde $y_{i}$ é o logaritmo da renda do filho da família, $x_{i}$ o logaritmo da renda do pai da família e $\varepsilon_{i}$ um termo de erro aleatório. O coeficiente $\beta$ mede a elasticidade intergeracional de renda e representa uma medida do grau de persistência intergeracional da renda. Um valor positivo do $\beta$ implica que um aumento de $1 \%$ na renda paterna está associado a um aumento médio de $\beta \%$ da renda dos filhos. Por exemplo, para um $\beta$ igual a 0,50 e uma renda paterna $30 \%$ acima da média de sua geração, implica uma renda do filho $15 \%$ acima da média da sua geração. A medida ( 1 - $\beta$ ) é chamada de grau de mobilidade intergeracional de renda. Quando $\beta$ é igual à unidade, a persistência intergeracional é máxima e a mobilidade intergeracional é nula. Quando $\beta$ é igual a zero, a persistência intergeracional é nula e a mobilidade intergeracional máxima 2,3.

Também se utilizou a abordagem das regressões quantílicas para mensuração de possíveis heterogeneidades na mobilidade intergeracional em função dos diferentes níveis de renda dos pais no passado. Ao invés de avaliar o efeito sobre a média do desfecho de interesse, a regressão quantílica avalia o efeito sobre os quantis da distribuição. As principais vantagens são: (i) fornece maior informação dos efeitos ao longo da distribuição, permitindo a comparação da mobilidade intergeracional entre várias classes de renda e (ii) possui a vantagem de não assumir qualquer linearidade na relação entre as rendas das gerações. Os quantis escolhidos são os seguintes: $10^{\circ}, 25^{\circ}$, $50^{\circ}, 75^{\circ}$ e $90^{\circ} 17,18$.

Como não possuímos a informação da renda do pai em diferentes pontos do tempo para estimar sua renda permanente, utilizamos a renda de um único ano (1984). Para tentar controlar as diferentes idades dos pais, essa variável foi incluída no modelo de regressão e, para controlar diferenças de saúde dos filhos ao nascer, foi incluído o escore $\mathrm{z}$ de peso ao nascer para idade gestacional.

\section{Resultados}

A Tabela 1 descreve a amostra estudada em termos de renda, escolaridade e idade dos filhos das famílias. Para os pais, em 1984, a média de renda foi de $\mathrm{R} \$ 428,00$, e em 1982 a média de escolaridade foi de 7,8 anos completos, e de idade foi de 30,9 anos. Ainda sobre a amostra resultante, vale destacar que em 1984 90,3\% e 98,9\% dos indivíduos tinham o pai e a mãe residindo com eles,

Tabela 1

Estatísticas descritivas dos filhos. Coorte de nascimentos de Pelotas, Rio Grande do Sul, Brasil, de 1982 a 2004/2005 $(\mathrm{N}=1.133)$

\begin{tabular}{|c|c|c|c|c|c|c|}
\hline \multirow[t]{2}{*}{ Variáveis } & \multicolumn{2}{|c|}{ Renda (Reais) } & \multicolumn{2}{|c|}{ Escolaridade (anos) } & \multicolumn{2}{|c|}{ Peso ao nascer (gramas) } \\
\hline & Média & Amplitude & Média & Amplitude & Média & Amplitude \\
\hline \multicolumn{7}{|l|}{ Sexo } \\
\hline Masculino & 632 & $52-20.305$ & 8,9 & $0-17$ & 3.273 & $1.450-4.850$ \\
\hline Feminino & 463 & $53-4.658$ & 10,2 & $0-17$ & 3.183 & $1.360-5.200$ \\
\hline \multicolumn{7}{|l|}{ Cor da pele } \\
\hline Branca & 595 & $53-20.305$ & 9,6 & $0-17$ & 3.258 & $1.450-5.200$ \\
\hline Preta/Parda & 399 & $52-1.530$ & 8,3 & $0-16$ & 3.125 & $1.360-4.740$ \\
\hline Todos & 562 & $52-20.305$ & 9,3 & $0-17$ & 3.236 & $1.360-5.200$ \\
\hline
\end{tabular}


respectivamente. Já em 2004, 74,6\% dos indivíduos residiam sem o pai e a mãe. Em 38,2\% dos domicílios resultantes em 2004, o próprio indivíduo era o chefe do domicílio, e em outros 14,5\% o chefe era o(a) cônjuge do indivíduo.

A Tabela 2 apresenta os resultados da estimação da persistência intergeracional para a subamostra completa. Na análise bruta, apresentaram-se regressões da renda dos filhos em função apenas da renda dos pais; na análise ajustada, foram adicionados o peso dos filhos ao nascer, o sexo e a cor da pele. Para as subamostras por sexo e cor da pele, na análise bruta, foram apresentadas regressões da renda dos filhos em função apenas da renda dos pais e na análise ajustada, adicionou-se apenas o peso dos filhos ao nascer.

$\mathrm{Na}$ amostra total, as estimativas da persistência entre as rendas dos filhos e dos pais são estatisticamente significativas, sendo 0,21 quando se considera apenas a renda do pai e 0,19 quando foram adicionados o peso, o sexo e a cor da pele. Para testar a existência de algum possível viés, dada a diferença de média de idade entre pais e filhos, foram estimadas as mesmas regressões para uma amostra composta por pais com idades entre 20 e 30 anos. Como os resultados não foram significativamente diferentes, eles não estão apresentados.

No caso do sexo, as estimativas da persistência entre as rendas dos filhos homens e a renda dos pais são todas estatisticamente significativas, sendo 0,20 quando se usa apenas a renda do pai e 0,19 quando se adiciona o peso ao nascer. As estimativas usando as rendas das filhas mulheres e a renda dos pais são todas estatisticamente significativas, sendo 0,24 quando se usa apenas a renda do pai ou quando se adicionou o peso ao nascer.
No caso da cor da pele, as estimativas da persistência entre as rendas dos filhos de cor branca e a renda dos pais são todas estatisticamente significativas, sendo 0,19 quando apenas a renda do pai é considerada e 0,18 quando se adiciona o peso ao nascer. As estimativas usando as rendas dos filhos de cor preta ou parda e a renda dos pais são todas estatisticamente significativas, sendo 0,21 quando se usa apenas a renda do pai e 0,19 quando se adiciona o peso ao nascer.

A Tabela 3 apresenta os resultados para as regressões quantílicas. Na amostra total, as estimativas da persistência intergeracional de renda são significativas em todos os quantis avaliados. Os resultados indicam uma forma de "U" para a persistência intergeracional de renda. Por exemplo, indivíduos que em 2004/2005 estavam entre os $10 \%$ mais pobres apresentam uma persistência de renda estimada em 0,29. Aqueles indivíduos localizados ao redor da mediana da distribuição a persistência estimada é em 0,11 . E, por fim, entre os $10 \%$ mais ricos o efeito da persistência é 0,30 . Quando se adiciona o peso ao nascer, constata-se a mesma relação, porém as estimativas da persistência são levemente inferiores. A Figura 1 apresenta as estimativas da persistência de renda pelos métodos de mínimos quadrados ordinários (MQO) e regressões quantílicas, tanto para análise bruta quanto para a análise ajustada. No gráfico, fica clara a forma de "U" que apresenta persistência de renda segundo as estimativas das regressões quantílicas.

$\mathrm{Na}$ análise por sexo, as estimativas ainda apontam para a presença do "U”, mas de forma menos acentuada. Para as mulheres, percebese que a persistência é maior especialmente entre aquelas mais pobres $(0,42)$, resultado bem

Tabela 2

Estimativas da persistência intergeracional de renda usando modelo de elasticidade. Coorte de nascimentos de Pelotas Rio Grande do Sul, Brasil, de 1982 a 2004/2005 ( $N=1.133$ ).

\begin{tabular}{|c|c|c|c|c|}
\hline \multirow[t]{2}{*}{ Variáveis } & \multicolumn{2}{|c|}{ Análise bruta } & \multicolumn{2}{|c|}{ Análise ajustada para peso ao nascer } \\
\hline & Coeficiente & Valor de $p$ * & Coeficiente & Valor de $p$ * \\
\hline \multicolumn{5}{|l|}{ Sexo } \\
\hline Masculino & 0,20 & $<0,01$ & 0,19 & $<0,01$ \\
\hline Feminino & 0,24 & $<0,001$ & 0,24 & $<0,01$ \\
\hline \multicolumn{5}{|l|}{ Cor da pele } \\
\hline Branca & 0,19 & $<0,01$ & 0,18 & $<0,01$ \\
\hline Preta/Parda & 0,21 & $<0,05$ & 0,19 & $<0,05$ \\
\hline Total & 0,21 & $<0,01$ & 0,19 & $<0,01$ \\
\hline
\end{tabular}

* Estimado pelo método mínimos quadrados ordinários. 
Tabela 3

Estimativas por regressões quantílicas da persistência intergeracional de renda usando o modelo de elasticidades. Coorte de nascimentos de Pelotas, Rio Grande do Sul, Brasil, de 1982 a 2004/2005 (N = 1.133).

\begin{tabular}{|c|c|c|c|c|c|c|}
\hline \multirow[t]{2}{*}{ Variáveis } & \multicolumn{2}{|c|}{ 及 10} & \multicolumn{2}{|c|}{ в 25} & \multicolumn{2}{|c|}{ 及 50} \\
\hline & Bruta & Ajustada & Bruta & Ajustada & Bruta & Ajustada \\
\hline \multicolumn{7}{|l|}{ Sexo } \\
\hline \multicolumn{7}{|l|}{ Masculino } \\
\hline Coeficiente & 0,14 & 0,16 & 0,09 & 0,09 & 0,14 & 0,13 \\
\hline Valor de $p$ * & 0,06 & 0,01 & 0,09 & 0,07 & 0,00 & 0,00 \\
\hline \multicolumn{7}{|l|}{ Feminino } \\
\hline Coeficiente & 0,42 & 0,42 & 0,28 & 0,28 & 0,16 & 0,17 \\
\hline Valor de $p$ * & 0,00 & 0,00 & 0,00 & 0,00 & 0,00 & 0,00 \\
\hline \multicolumn{7}{|l|}{ Cor da pele } \\
\hline \multicolumn{7}{|l|}{ Branca } \\
\hline Coeficiente & 0,26 & 0,28 & 0,09 & 0,10 & 0,11 & 0,12 \\
\hline Valor de $p$ * & 0,00 & 0,00 & 0,02 & 0,02 & 0,00 & 0,00 \\
\hline \multicolumn{7}{|l|}{ Não branca } \\
\hline Coeficiente & 0,54 & 0,51 & 0,20 & 0,15 & 0,16 & 0,14 \\
\hline Valor de $p$ * & 0,02 & 0,04 & 0,24 & 0,30 & 0,05 & 0,13 \\
\hline \multicolumn{7}{|l|}{ Total } \\
\hline Coeficiente & 0,29 & 0,23 & 0,12 & 0,12 & 0,11 & 0,14 \\
\hline Valor de $p$ * & 0,00 & 0,00 & 0,00 & 0,00 & 0,00 & 0,00 \\
\hline \multirow[t]{2}{*}{ Variáveis } & \multicolumn{2}{|c|}{$\beta 75$} & \multicolumn{2}{|c|}{ 及 90} & & \\
\hline & Bruta & Ajustada & Bruta & Ajustada & & \\
\hline \multicolumn{7}{|l|}{ Sexo } \\
\hline \multicolumn{7}{|l|}{ Masculino } \\
\hline Coeficiente & 0,21 & 0,21 & 0,36 & 0,31 & & \\
\hline Valor de $p$ * & 0,00 & 0,00 & 0,00 & 0,00 & & \\
\hline \multicolumn{7}{|l|}{ Feminino } \\
\hline Coeficiente & 0,17 & 0,18 & 0,24 & 0,24 & & \\
\hline Valor de p * & 0,00 & 0,00 & 0,00 & 0,00 & & \\
\hline \multicolumn{7}{|l|}{ Cor da pele } \\
\hline \multicolumn{7}{|l|}{ Branca } \\
\hline Coeficiente & 0,19 & 0,19 & 0,34 & 0,31 & & \\
\hline Valor de $p^{*}$ & 0,00 & 0,00 & 0,00 & 0,00 & & \\
\hline \multicolumn{7}{|l|}{ Não branca } \\
\hline Coeficiente & 0,18 & 0,16 & 0,26 & 0,26 & & \\
\hline Valor de $p$ * & 0,04 & 0,03 & 0,05 & 0,08 & & \\
\hline \multicolumn{7}{|l|}{ Total } \\
\hline Coeficiente & 0,21 & 0,19 & 0,30 & 0,25 & & \\
\hline Valor de $p$ * & 0,00 & 0,00 & 0,00 & 0,00 & & \\
\hline
\end{tabular}

* Estimado a partir das regressões quantílicas.

diferente daquele observado para os homens em que a persistência é mais forte entre aqueles com maior renda $(0,36)$.

Resultado similar ao obtido por sexo é o observado por cor. Entre os brancos a persistência é maior entre os mais ricos, 0,34 , enquanto entre os não brancos observa-se a maior estimativa de persistência entre os $10 \%$ mais pobres 0,54 . Em ambas as amostras, sexo e cor, as estimativas ajustadas pelo peso ao nascer são similares às verificadas nas análises brutas, com a ressalva de apresentarem levemente menores magnitudes. 
Comparativo das estimativas da persistência intergeracional de renda usando o modelo de elasticidades pelos métodos de MQO (mínimo quadrado ordinário) e regressões quantílicas. Coorte de nascimentos de Pelotas, Rio Grande do Sul, Brasil, de 1982 a 2004/2005 ( $N=1.133)$.

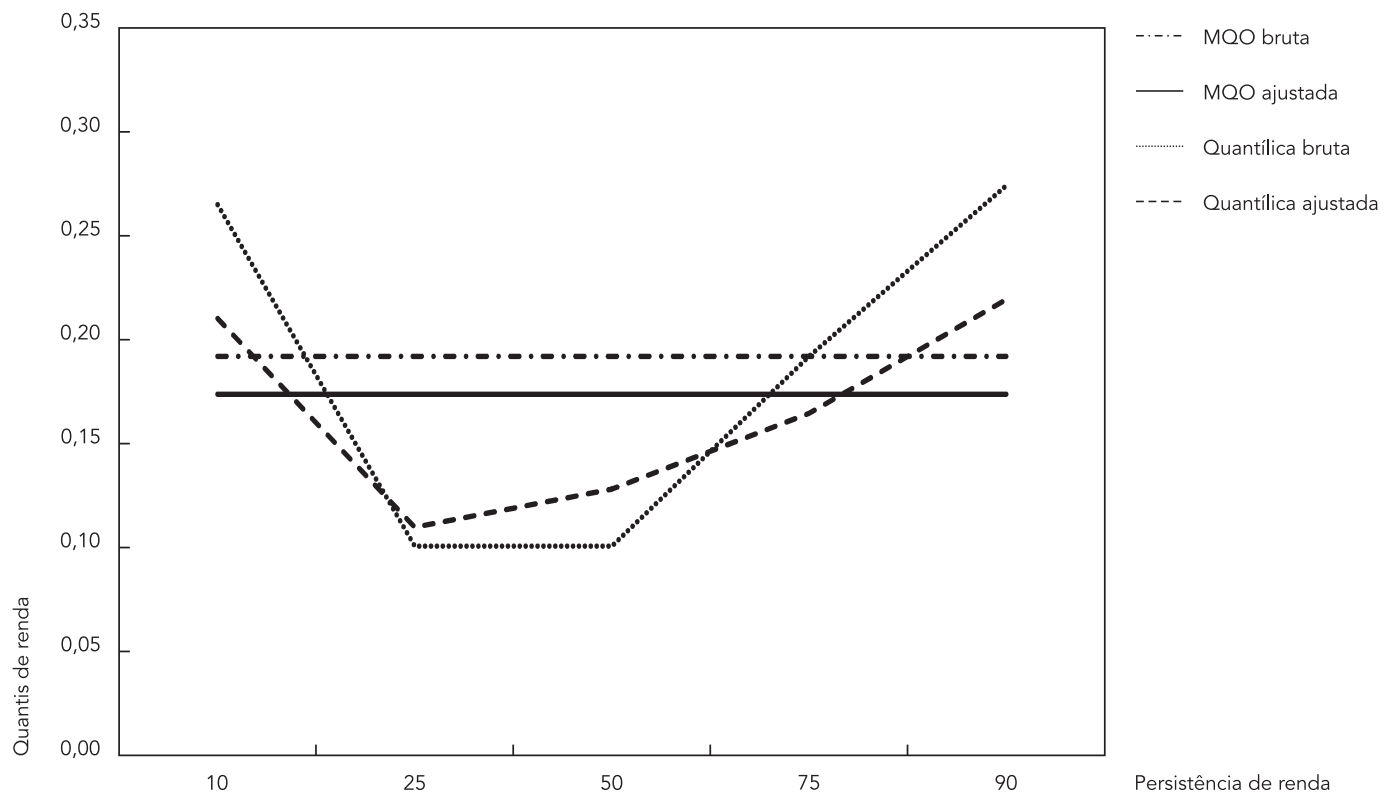

\section{Discussão}

São poucos os trabalhos que estimam mobilidade intergeracional da renda no Brasil. Uma boa justificativa para essa escassez de trabalhos está na dificuldade de se obter informações de pais e filhos para a estimação da mobilidade usando a equação ${ }^{1}$. Os trabalhos existentes no Brasil estão concentrados em informações das PNAD de 1982, 1988 e 1996 ou, no Censo Demográfico de 2000. Essas bases de dados não contêm informação de renda dos pais e dos filhos. Apesar dessas dificuldades, esses estudos, estimando indiretamente a elasticidade intergeracional de renda, concluem que o Brasil apresenta uma das maiores persistências intergeracionais de renda do mundo 13,14 16,19,20.

O presente estudo contribui para o debate ao apresentar o caso de uma cidade de médio porte, Pelotas. Os resultados encontrados apresentam uma persistência estimada relativamente baixa. As estimativas da elasticidade intergeracional de renda variam entre 0,19 e 0,21 . As estimativas obtidas pelas regressões quantílicas se situam em um intervalo bem maior 0,09 e 0,54. Ainda assim, estudos encontram para o Brasil elasticidades muito maiores: um deles encontrou elasticidades variando entre 0,67 e 0,80 , outro entre 0,53 e 0,69 e um terceiro encontrou uma elasticidade de $0,6813,16,20$.

As explicações para o baixo valor encontrado no trabalho podem ser agrupadas em dois tipos. Primeiro grupo, relacionado a aspectos estatísticos. Quando são utilizados dados da renda de um único ano como uma medida da renda permanente, há uma subestimação do coeficiente $8,21,22,23$. Esse estudo dispunha de dados de um único ano de renda do filho e, portanto, é esperada uma estimativa da persistência abaixo da real. Adicionalmente, há consenso na literatura de que o ponto de observação afeta as estimativas da persistência intergeracional de renda. Estudos mostram que quando os dados de renda dos filhos são coletados em idades muito jovens, o coeficiente tende a ser relativamente baixo $8,21,22,23$. Neste estudo a média das idades dos filhos ficou em torno dos 23 anos, logo é plausível esperar que a renda obtida em 2004/2005 não constitua ainda a renda permanente do filho. De fato, um estudo brasileiro usando renda dos filhos em torno dos 20 anos encontrou uma elasticidade de 0,42 13. Resultados similares têm sido 
encontrados em estudos para Cingapura, usando dados de jovens com idades entre 23 e 29 anos, Estados Unidos em torno dos 20 anos, Malásia em torno de 25 anos, e Alemanha em torno de 23 anos 24,25,26,27. Contudo, uma vantagem do trabalho é que são utilizados dados de renda e não estimações indiretas dela como fazem os outros trabalhos sobre o Brasil.

O segundo grupo de explicações tenta mostrar que realmente aumentou a mobilidade intergeracional de renda no Brasil nos últimos anos. Os trabalhos de referência para mobilidade intergeracional de renda do Brasil estão baseados em banco de dados dos anos 1990, particularmente da PNAD de 1996. Assim, a posição socioeconômica dos filhos é da década de 1990 e a dos pais é referente a décadas anteriores. Este estudo utiliza dados dos filhos em 2004/2005. O Brasil passou por importantes transformações políticas, econômicas, demográficas e sociais nas últimas décadas 28 que podem ter influenciado o grau da mobilidade intergeracional de renda.

Foi visto que, teoricamente, quatros fatores influenciam esse grau. Primeiro, maior será o grau de mobilidade intergeracional de renda quanto menor o investimento privado dos pais no capital humano dos filhos. Tal fator parece não ter influenciado na redução do grau de mobilidade. Os gastos das famílias com educação aumentaram a sua participação no total de despesas correntes entre 1987-1988 e 2002-2003, passando de $3,2 \%$ para $5,5 \%$, respectivamente. Paralelamente, houve um aumento da participação dos gastos com cursos regulares (cursos préescolares e cursos regulares dos ensinos Fundamental, Médio e Superior) e outros cursos nas despesas com educação das famílias 29.

Segundo, maior será o grau de mobilidade intergeracional de renda quanto menor o retorno para qualquer investimento em capital humano. Diversas pesquisas têm analisado a relação entre educação e salários no Brasil. Várias delas encontraram altas taxas de retorno à educação no Brasil: um ano a mais de estudo gera, em média, uma taxa de retorno de $15 \%$ ou mais 30,31 . No entanto, uma pesquisa mais recente 32 , usando dados do período de 1997 a 2007, constata que esse retorno varia entre $11 \%$ e $5,5 \%$. Ou seja, o retorno dos investimentos em educação parece ter se reduzido ao longo do tempo com consequente aumento da mobilidade intergeracional de renda.

Terceiro, maior será o grau de mobilidade intergeracional de renda quanto menor o grau de transmissão de outros aspectos do background familiar importantes para os rendimentos. Um estudo 13 demonstrou que a mobilidade intergeracional de renda no Brasil tem aumentado na segunda metade do século passado como resul- tado do aumento na mobilidade intergeracional de educação. Outro estudo ${ }^{33}$, realizado na cidade de Recife, Pernambuco, mostra que a mobilidade intergeracional de educação tem aumentado entre os anos de 1996 e 2010.

Finalmente, maior será o grau de mobilidade intergeracional de renda quanto mais a favor dos mais pobres seja o investimento público em capital humano. Desde meados da década de 1990, houve um avanço de vários indicadores educacionais no Brasil. Na prática, nesse período foi universalizado o acesso ao ensino fundamental e houve uma grande expansão do Ensino Médio 34. Por exemplo, a porcentagem de pessoas com ensino médio completo foi de cerca de $20 \%$ na geração nascida em 1960, 25\% na de 1970 e aumentou expressivamente para 50\% no caso da geração nascida em 198235.

Por todas essas razões, existe uma expectativa de que o Brasil apresente maiores graus de mobilidade intergeracional do que aqueles apresentados pelos trabalhos baseados em informações das décadas passadas. Por fim, uma última possível explicação deve estar na heterogeneidade regional existente em um país com dimensão continental, como é o caso do Brasil. Os estudos existentes tratam da mobilidade de renda do país e, por isso, estimam um comportamento médio. Este estudo utilizou dados da coorte de Pelotas, e a análise dos resultados deve levar em conta esse fato.

As análises das regressões quantílicas reforçam o resultado obtido pela estimação da elasticidade intergeracional da renda. Além disso, indicam que a maior mobilidade social acontece nas camadas sociais intermediárias. Os resultados encontrados reforçam a existência de dois polos opostos de armadilhas: armadilha da pobreza e da riqueza. Em parte, o fato de as categorias extremas possuírem maior dependência de renda pode apenas representar o fato de que tais categorias possuem apenas um sentido de movimento. Diferente de quem está posicionado nas categorias intermediárias, os indivíduos posicionados nos extremos podem se mover somente para uma categoria inferior ou somente para uma categoria superior. De mais concreto, a análise das regressões quantílicas reforça um resultado já conhecido na literatura de mobilidade intergeracional de renda: não existe linearidade na relação entre a renda do pai e a renda do filho.

Se de um lado o Brasil ainda é um país com uma elevada desigualdade social, este estudo apresentou uma luz no final do túnel, de uma mudança de comportamento que pode indicar o início de um processo de maior mobilidade de renda que resulte em uma sociedade menos desigual. 


\section{Resumen}

Brasil está considerado como uno de los países con menor movilidad intergeneracional de ingresos del mundo. El principal objetivo de este estudio es examinar la movilidad intergeneracional de ingresos en la cohorte de nacimientos de 1982 en Pelotas, Rio Grande do Sul. Se utilizan dos métodos para la estimación: la elasticidad intergeneracional de los ingresos y regresiones por cuartiles de ingresos para los diferentes niveles de ingresos de los padres en el pasado. Los resultados sugieren una movilidad intergeneracional de ingresos relativamente alta para los estándares brasileños. La explicación radica principalmente en el hecho de que fueron utilizados datos de ingresos de los hijos cuando eran muy jóvenes, alrededor de 23 años. Los análisis de regresiones por cuartiles indican que una mayor movilidad social se produce en las capas intermedias. Los resultados refuerzan la existencia de dos polos opuestos: el de la pobreza y el de la riqueza.

Clase Social; Renta; Estudios de Cohortes

\section{Colaboradores}

C. A. O. Tejada, A. D. Bertoldi, A. Carraro, J. V. S. Motta e A. J. D. Barros foram os responsáveis pelo desenho do projeto, redação, revisão crítica de literatura relevante e de conteúdo intelectual, análise e interpretação de dados e aprovação da versão final. F. G. Ribeiro, F. C. Barros e B. L. Horta forma responsáveis pela revisão crítica de conteúdo intelectual, análise e interpretação de dados e aprovação da versão final.

\section{Agradecimentos}

Este artigo é baseado em dados da coorte de nascimentos de 1982 em Pelotas que atualmente é apoiado pela Iniciativa Wellcome Trust intitulada Major Awards for Latin America on Health Consequences of Population Change. Fases anteriores do estudo foram apoiadas pelo International Development Research Center, a Organização Mundial da Saúde, Overseas Development Administration, União Europeia, Programa Nacional de Apoio a Núcleos de Excelência, CNPq e Ministério da Saúde.

\section{Referências}

1. Ferreira FH, Messina J, Rigolini J, López-Calva L-F, Lugo MA, Vakis R. Economic mobility and the rise of the Latin American middle class. Washington DC: The World Bank Group; 2013.

2. Azevedo VM, Bouillon CP. Intergenerational social mobility in Latin America: a review of existing evidence. Revista de Análisis Económico 2010; 25: 7-42.

3. Corak M. Generational income mobility in North America and Europe: an introduction. In: Corak M, editor. Generational income mobility in North America and Europe. New York: Cambridge University Press; 2004. p. 1-37.

4. Solon G. Intergenerational mobility in the labor market. In: Card D, Ashenfelter OC, editors. Handbook of labor economics. Amsterdam: Elsevier; 1999. p. $1761-800$
5. Becker GS, Tomes N. An equilibrium theory of the distribution of income and intergenerational mobility. J Polit Econ 1979; 87:1153-89.

6. Solon G. A model of intergenerational mobility variation over time and place. In: Corak M, editor. Generational income mobility in North America and Europe. New York: Cambridge University Press; 2004. p. 38-47.

7. Black SE, Devereux PJ. Recent developments in intergenerational mobility. In: Card D, Ashenfelter O, editors. Handbook of labor economics. Amsterdam: Elsevier; 2011. p. 1487-541.

8. Solon G. Cross-country differences in intergenerational earnings mobility. J Econ Perspect 2002; 16:59-66.

9. Dearden L, Machin S, Reed H. Intergenerational mobility in Britain. Econ J (London) 1997; 107: 47-66. 
10. Mocetti S. Intergenerational earnings mobility in Italy. B E J Econom Anal Policy 2007; 7:1794-819.

11. Piraino P. Comparable estimates of intergenerational income mobility in Italy. B E J Econom Anal Policy 2007; 7:1711-38.

12. Ueda A. Intergenerational mobility of earnings and income in Japan. B E J Econom Anal Policy 2009; 9:2203-30.

13. Dunn CE. The intergenerational transmission of lifetime earnings: evidence from Brazil. B E J Econom Anal Policy 2007; 7:1782-93.

14. Andrade E, Veloso F, Madalozzo R, Ferreira S. Do borrowing constraints decrease intergenerational mobility in Brazil? A test using quantile regression. São Paulo: Insper; 2003. (Working Paper, 2003-3).

15. Checchi D, Dardanoni V. Mobility comparisons: does using different measures matter? In: Amiel Y, Bishop JA, editors. Inequality, welfare and poverty: theory and measurement. Bingley: Emerald Group Publishing Limited; 2003. p. 113-45.

16. Ferreira SG, Veloso FA. Intergenerational mobility of wages in Brazil. Revista Brasileira de Econometria 2006; 26:181-211.

18. Buchinsky M. Recent advances in quantile regression models: a practical guideline or empirical research. J Hum Res 1998; 33:88-126.

18. Koenker R, Basset Jr. G. Quantile regression. Econometrica 1978; 46:33-50.

19. Netto Junior JLDS, Ramalho HMDB, Aragón EKDSB. Transmissão intergeracional de educação e mobilidade de renda no Brasil. Economia e Desenvolvimento 2013; 2:6-34.

20. Pero V, Szerman D. Mobilidade intergeracional de renda no Brasil. Pesquisa e Planejamento Econômico 2008; 38:1-35.

21. Grawe ND. Lifecycle bias in estimates of intergenerational earnings persistence. Labour Econ 2006; 13:551-70

22. Haider S, Solon G. Life-cycle variation in the association between current and lifetime earnings. Am Econ Rev 2006; 96:1308-20.

23. Mazumder B. Fortunate sons: new estimates of intergenerational mobility in the United States using social security earnings data. Rev Econ Stat 2005; 87:235-55
24. Couch KA, Dunn TA. Intergenerational correlations in labor market status: a comparison of the United States and Germany. J Hum Res 1997; 32:210-32.

25. Lillard LA, Kilburn MR. Intergenerational earnings links: sons and daughters. Santa Monica: RAND Corporation; 1995. (Working Paper Series, 95-17).

26. Ng I. Intergenerational income mobility in Singapore. B E J Econom Anal Policy 2007; 7:1713-8.

27. Black SE, Devereux PJ. Recent developments in intergenerational mobility. Cambridge: National Bureau of Economic Research; 2010. (Working Paper, 15889).

28. Paim J, Travassos C, Almeida C, Bahia L, Macinko J. The Brazilian health system: history, advances, and challenges. Lancet 2011; 377:1778-97.

29. Castro J, Vaz F. Gastos da família com educação. In: Silveira FG, Servo LM, Menezes T, Piola SF, organizadores. Gasto e consumo das famílias brasileiras contemporâneas. Brasília: Instituto de Pesquisa Econômica Aplicada; 2007. p. 77-104

30. Barbosa Filho FH, Pessôa S. Retorno da educação no Brasil. Pesquisa e Planejamento Econômico 2008; 38:97-125.

31. Resende M, Wyllie R. Retornos para educação no Brasil: evidências empíricas adicionais. Econômica Aplicada 2006; 10:349-65.

32. Texeira WM, Menezes-Filho NA. Estimando o retorno à educação do Brasil considerando a legislação educacional brasileira como um instrumento. Revista de Economia Política 2012; 32:479-96.

33. Gonçalves MB, Silveira Neto RM. Persistência intergeracional de educação no Brasil: o caso da Região Metropolitana do Recife. Estudos Econômicos 2013; 43:432-63.

34. Veloso F. A evolução recente e propostas para a melhoria da educação no Brasil. In: Bacha EL, Schwartzman S, organizadores. Brasil: a nova agenda social. Rio de Janeiro: LTC; 2011. p. 215-53.

35. Menezes-Filho NA. Os determinantes do desempenho escolar do Brasil. São Paulo: Instituto Futuro Brasil; 2007.

Recebido em 01/Mai/2014

Versão final reapresentada em 29/Set/2014

Aprovado em 09/Jan/2015 